\title{
Priorización de Activos Físicos centrado en el Rendimiento Global (Throughput) en una Planta de Chancado
}

\author{
Orlando M. Durán ${ }^{1}$, Vicente González-Prida ${ }^{2}$, Adolfo Crespo ${ }^{2}$ y Antonio Guillén ${ }^{2}$ \\ (1) Escuela de Ingeniería Mecánica, Pontificia Universidad Católica de Valparaíso. Valparaíso, Chile. \\ (Correo-e: orlando.duran@pucv.cl) \\ (2) Universidad de Sevilla, Sevilla. España. (Correo-e: vicente.gonzalezprida@gdels.com; ajguillen@us.es; \\ adolfo@us.es).
}

Recibido Jun. 1, 2018; Aceptado Ago. 6, 2018; Versión final Oct. 26, 2018, Publicado Abr. 2019

\section{Resumen}

Se propone una técnica para la priorización de activos físicos que se basa en la contribución que hace cada uno de ellos al rendimiento global (throughput). La técnica presentada está basada en la Eficiencia del Rendimiento Global del Sistema (Overall Throughput Effectiveness, OTE) y relaciona la disponibilidad de cada equipo con su utilización y con el rendimiento global del sistema. La hipótesis es que, bajo la óptica del mantenimiento se pueden jerarquizar equipos en función del impacto que su indisponibilidad genera en el desempeño a nivel de fábrica. A través de un estudio de caso, basado en una planta de conminución chilena, se demuestra la viabilidad de la técnica y la posibilidad de analizar las tendencias que los impactos de los equipos muestran en el tiempo. Se concluye que la técnica puede ser utilizada para definir las políticas de mantenimiento, destinar recursos y revisar decisiones operativas, tales como mejorar los niveles de utilización y disponibilidad.

Palabras clave: disponibilidad; gestión de activos; productividad; OTE

\section{Throughput based Prioritization of Physical Assets in a Crushing Plant}

\begin{abstract}
A physical assets prioritization technique is proposed. The technique is based on the contribution that each equipment makes to global throughput. The proposed technique is based on the Overall Throughput Effectiveness (OTE) and relates the availability of each equipment, its utilization index with the throughput of the entire system. The hypothesis is that, from the point of view of maintenance, equipment can be ranked according to the impact that their unavailability generates in the performance at the factory level. Through a case study, based on a Chilean comminution plant, the viability of the technique and the possibility of analyzing the trends that the equipment's impacts show over time are demonstrated. It is concluded that the technique can be used to define maintenance policies, allocate resources and review operational decisions, in terms of improving levels of use and availability.
\end{abstract}

Keywords: availability; throughput; assets management; productivity; O.T.E. 


\section{INTRODUCCIÓN}

En escenarios actuales, los sistemas productivos son cada vez más complejos y con equipos cada vez más sofisticados y caros. La realidad exige una reducción continua en los costos y la maximización de los niveles de producción (Silva et al., 2014). Asimismo, los presupuestos para el mantenimiento son cada vez más restringidos y la segmentación y priorización de los activos es una tarea fundamental y necesaria. Según Durán (2003), el éxito de la implantación de sistemas de mantenimiento eficientes depende de muy buenos estudios de criticidad y de la búsqueda de potenciales para lograr así, retornos económicos y productivos. Aún más (Maior et al., 2016), un adecuado nivel de conocimiento de la confiabilidad y del desempeño de la función mantenimiento traerá ventajas competitivas a toda firma. El modelo de ciclo para la gestión de mantenimiento desarrollado por Crespo (2007) incluye, precisamente la priorización de activos como la segunda fase de este ciclo. Son varios los enfoques que pueden emplearse para jerarquizar equipos buscando la optimización de la gestión de activos. Las técnicas para la priorización de activos físicos más utilizadas son los Diagramas de Pareto, Jack Knife y los métodos multicriteriales (MCDM), tales como el A.H.P y Delphi. También es importante recordar que el análisis de criticidad constituye una fase fundamental del RCM y del FMECA (Gasca et al. , 2017). En ellos, se emplea el Número de Prioridad de Riesgo (Risk Priority Number - RPN) como índice multicriterio de evaluación (Crespo y Parra, 2012). La tendencia es emplear modelos que tengan en cuenta los aspectos claves para la evaluación y que están directamente conectados con los objetivos del negocio y la generación de valor. En este trabajo se intenta hacer una contribución en este sentido.

En la mayoría de estas técnicas se introduce de una u otra forma la disponibilidad, o la indisponibilidad, como uno de los factores claves en la evaluación. Kristjanpoller et al. (2015) propone una metodología llamada RAM-C, la que permite conocer el efecto de cada equipo sobre el sistema que lo contiene incluyendo situaciones donde la configuración lógica no está en dependencia de serie. Esta metodología establece una jerarquía entre los activos de un sistema productivo usando como base el llamado Factor de Impacto de cada uno de ellos. Para el cálculo de este índice se lleva en consideración la disponibilidad y configuración de los equipos, pero no el efecto que la pérdida de disponibilidad podrá generar en las tasas de producción a nivel de sistema. Se aprecian dos aspectos a mejorar en el enfoque de los problemas de jerarquización: (i) la indisponibilidad se suele tratar como un efecto local, a nivel del equipo, sin considerar la repercusión sobre el sistema en el que el equipo está integrado; (ii) no se evalúa el efecto que la indisponibilidad tiene sobre la producción. Para emplear la indisponibilidad como criterio, esta debe implicar una pérdida de producción, o sea, indisponibilidad que se traduzca en producir menos.

La jerarquización de equipos exige la "comparación" entre ellos. Dicha comparación ha de hacerse necesariamente desde un nivel de análisis superior al del equipo, con una visión del conjunto. Se tendrá entonces, dos niveles de análisis de la indisponibilidad: (i) local o nivel de equipo, (ii) sistémica o a nivel sistema. En este sentido, un equipo será más crítico si su indisponibilidad genera a su vez la indisponibilidad del sistema (Crespo et al. 2016). En muchos casos las indisponibilidades locales no tienen consecuencias sobre la producción a nivel de sistema. Esto debería tener un reflejo en la planificación del mantenimiento. Es el caso, por ejemplo, de un evento de indisponibilidad en equipos redundados. Es esta redundancia la que evita que la indisponibilidad de un equipo se traslade al sistema. Esto ha de llevar a que el equipo tenga una criticidad menor y, consecuentemente, un plan de mantenimiento ajustado que tenga en cuenta esta situación. Sin embargo, en escenarios industriales reales no se llega a evaluar la pérdida de producción real provocada por un evento de indisponibilidad.

Los sistemas de producción intensiva, como puede ser la extracción minera, se caracterizan porque si un equipo crítico falla, ocurrirá un efecto negativo en los rendimientos globales de producción (throughput). Tanto más negativo es este impacto mayor será la criticidad del equipo. Es decir, la criticidad está íntimamente relacionada con la generación de valor (Espinoza y Cea, 2004). Pascual et al. (2011) proponen un método para priorizar equipos usando una técnica llamada Diagramas de Influencia de la Eficiencia del Sistema (System Efficiency Influence Diagram - SEID) la cual permite identificar los equipos de una línea de producción que generan el mayor impacto en las tasas de producción de manera tal a definir las políticas de mantenimiento apropiadas. Luego, Pascual et al. (2016) aplica dicha metodología como un procedimiento iterativo de optimización basándose en el rendimiento del sistema productivo, a través de conceptos de la teoría de restricciones y detección de cuellos de botella.

No todos los equipos pertenecientes a un sistema productivo, sin importar la estructura a través de la cual están relacionados entre ellos, hacen la misma contribución al rendimiento del sistema. Dicho de otra forma, el rendimiento del sistema no es sensible de la misma forma a los diferentes desempeños de los equipos. Diferentes niveles de disponibilidad, utilización, y calidad, etc., hacen que cada equipo tenga diferentes niveles de contribución al rendimiento global del sistema. Esto significa que no solo la disponibilidad de cada equipo determinará la importancia de este, sino una combinación entre esta y los niveles de utilización de 
cada uno de ellos llevando en cuenta la taxonomía del sistema productivo. Este aspecto no ha sido correctamente llevado en consideración por los modelos mencionados anteriormente.

Generar modelos que puedan dar lugar a métodos de evaluación cuantitativos y que reflejen de manera directa aspectos de alto impacto para la gestión de la organización sigue siendo un reto. Los modelos basados en indicadores son especialmente interesantes ya que permiten la automatización del proceso de evaluación a partir de la parametrización de estos modelos como parte de paquetes de software de apoyo al mantenimiento. Este tipo de enfoque apunta a modelos de mantenimiento más inteligentes y dinámicos que son los que demanda en el modelo de Industria 4.0. En este trabajo se propone un modelo de evaluación de la criticidad a partir del uso del indicador de eficiencia del rendimiento global (OTE). EI OTE, propuesto por Muthiah (2007), permite detectar cuellos de botella en sistemas productivos según su configuración. El OTE llena un vacío existente dejado por el uso del OEE (Nakajima, 1988) que solo es capaz de evaluar la eficiencia a nivel de equipo, pero no a nivel de sistemas productivos. Más adelante, Muthiah (2008) presenta un mecanismo automatizado para la detección de cuellos de botella. El modelo de evaluación propuesto en este trabajo combina el uso del OTE con los niveles de utilización de los equipos para definir prioridades entre activos.

\section{INDICADORES DE EFICIENCIA}

En esta sección se describen dos indicadores de eficiencia, la Efectividad Global del Equipo (Overall Equipment Effectiveness, O.E.E) y la Efectividad Global de la Producción (Overall Throughput Effectiveness, de aquí en adelante O.T.E.). Se presentan sus formulaciones y se analizan sus ventajas y defectos.

Efectividad Global del Equipo (Overall Equipment Effectiveness, OEE). El OEE, según Nakajima (1988), puede ser calculado a través del producto entre la Eficiencia de la Disponibilidad (Aeff), el desempeño productivo (Peff), esto es, descontadas las pérdidas de ritmo o de productividad, las detenciones y esperas, y, la eficiencia de la calidad (Qeff ), descontados de ella las pérdidas por defectos y por retrabajo. O sea:

OEE $=$ Aeff * Peff * Qeff

Este índice se remite a calcular la eficiencia a nivel de equipo y mide la productividad del equipo en relación con su máxima capacidad, que se supone constante durante el período de tiempo usado para medir el desempeño, sin proporcionar una visión global a nivel de sistema productivo, ni menos el impacto de equipos específicos en el desempeño global. Esta insuficiencia del OEE ha llevado a modificaciones para adecuarse a una perspectiva a nivel de sistema productivo. Con la modificación de OEE, diferentes índices han sido sugeridos en la literatura. Entre las formulaciones propuestas se puede mencionar: Efectividad Global del Proceso (Overall Process Effectiveness), Efectividad Total del Proceso (Total Overall Process Effectiveness o TOPE) y Efectividad Global de la Producción (OTE), (Raja y Kannan, 2010; Lanza et al., 2013; Alsyouf, 2001).

\section{Efectividad Global de la Producción (Overall Throughput Effectiveness, OTE)}

A fin de abordar la limitación mencionada en el capítulo anterior y considerando que todo sistema de producción puede poseer diversas configuraciones entre sus equipos (serie y paralelo entre los más usados (Sellito y Brusius, 2017), el OTE fue desarrollado por Muthiah y Huang (2007). Tal métrica se define teniendo en cuenta que coexisten configuraciones diversas de subsistemas, incluyendo serie, en paralelo, de montaje y de expansión (Figura 1). El propósito de la OTE es doble: la medición de rendimiento a nivel de fábrica y la realización de acciones de diagnóstico (también a nivel de fábrica), tales como la detección de cuellos de botella y la identificación de capacidad oculta. El OTE se desarrolla en base a la comparación de la productividad real y de la productividad máxima alcanzable, que es la misma idea detrás del desarrollo de OEE. Teniendo en cuenta que la OEE en la ecuación (1) se puede simplificar aún más como:

O. E. E. $=\frac{\text { Rendimiento Real del Equipo (unidades)en el tiempo total }}{\text { Rendimiento Teórico del Equipo (unidades)en el tiempo total }}$

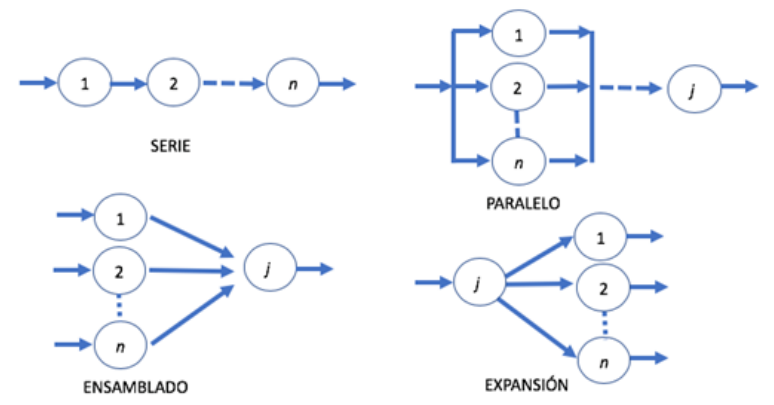

Fig. 1: Alternativas de configuración para la composición del O.T.E. 
Al extender esta definición a nivel de fábrica, se tiene:

O.T.E. $=\frac{\text { Rendimiento Real del Sistema (unidades) en el tiempo total }}{\text { Rendimiento Teórico del Sistema (unidades) en el tiempo total }}$

Existe un conjunto de modelos de OTE para cada una de las configuraciones de los subsistemas ya mencionadas. La Tabla 1 presenta las métricas desarrolladas. La idea es la siguiente. Si una parte necesita ser procesada por el equipo de $A$ antes de que pueda ser procesada por el equipo B (por ejemplo, $A$ y $B$ están conectados en serie), entonces la cantidad de salida buena del producto desde el equipo B está limitado por los productos buenos que salieron del equipo $A$.

Tabla 1: Métricas OTE desarrolladas según diferentes Configuraciones.

\begin{tabular}{|c|c|}
\hline Subsistemas & O.T.EE \\
\hline Serie & $\frac{\min \left\{\min _{i=1,2, \ldots n-1}\left\{O E E_{(i)} \times R_{t h(i)} \times \prod_{j=i+1}^{n} Q_{e f f(j)}\right\}, O E E_{(n)} \times R_{t h(n)}\right\}}{\min _{i=1,2, \ldots, n}\left\{R_{t h(i)}\right\}}$ \\
\hline Paralelo & $\frac{\sum_{i=1}^{n}\left(O E E_{(i)} \times R_{t h(i)}\right)}{\sum_{i=1}^{n} R_{t h(i)}}$ \\
\hline Unión & $\frac{\min \left\{\min _{i=1,2, \ldots n-1}\left\{O E E_{(i)} \times\left(R_{\operatorname{th}(i)} / k_{A(i)}\right) \times Q_{e f f(a)}\right\}, R_{t h(a)} \times O E E_{(a)}\right\}}{\min \left\{\min _{i=1,2, \ldots n-1}\left\{\left(R_{\operatorname{th}(i)} / k_{A(i)}\right) \times Q_{e f f(a)}\right\}, R_{t h(a)}\right\}}$ \\
\hline Expansion & $\frac{\sum_{i=1}^{n} \min \left(R_{t h(e)} \times O E E_{(e)} \times k_{E(i)} \times Q_{e f f(i)}, R_{t h(i)} \times O E E_{(a)}\right)}{\sum_{i=1}^{n} \min \left\{R_{t h(e)} \times k_{E(i)}, R_{t h(i)}\right\}}$ \\
\hline
\end{tabular}

Asumiendo que el equipo $i$ procesa $n$ productos diferentes. Sea $Q_{\text {eff(ij) }}$ y $R_{\text {th(ij) }}$ la eficiencia de calidad y la tasa de procesamiento teórica del equipo $i$ al procesar el producto $j(j=1,2, \ldots, n)$, respectivamente. Sea $X_{(i j)}$ el porcentaje de mezcla del producto $j$ en el equipo $i\left(Q_{e f f}\right.$ (i) y $R_{\text {th(i) }}$ para múltiples productos se puede calcular como sigue:

$$
\begin{aligned}
& \mathrm{Q}_{\text {eff(i) }}=\sum_{\mathrm{j}=1}^{\mathrm{n}}\left(\mathrm{X}_{(\mathrm{ij})} \mathrm{XQ}_{\mathrm{eff}(\mathrm{ij})}\right) \\
& \mathrm{R}_{\mathrm{th}(\mathrm{i})}=\sum_{\mathrm{j}=1}^{\mathrm{n}}\left(\mathrm{X}_{(\mathrm{ij})} \mathrm{x} \mathrm{R}_{\mathrm{th}(\mathrm{ij})}\right)
\end{aligned}
$$

\section{METODOLOGIA}

Uno de los mayores desafíos para identificar oportunidades en procesos de mejoría en gestión de activos es establecer priorizaciones realistas y "automatizables". Uno de los principales puntos en estos análisis es poder saber cuál es el efecto que cada elemento de un sistema genera en el rendimiento global del mismo (Visión de negocio). Cuanto más compleja es la estructura, más difícil es determinar este efecto, y menos relevante se presentan aquellos análisis basados únicamente en la confiabilidad. Desde el punto de vista de la priorización, no todos los equipos tienen la misma importancia, pues no todos los activos, cuando indisponibles, provocarán el mismo "daño" al negocio. O sea, hay equipos ante los cuales el sistema es más sensible que otros en cuanto a la pérdida de rendimiento global cuando se verifican variaciones en la disponibilidad y/o en la utilización de este (Figura 2 Caso A). Por otra parte, existen equipos, donde se verifican menores efectos (menor sensibilidad) en el OTE al sufrir variaciones en su disponibilidad y o en la utilización (Figura 2 Caso B). Dicho de otra forma, hay equipos, que de acuerdo con la configuración en que se encuentran en el sistema productivo, el rendimiento será más o menos sensible a variaciones en sus respectivos valores de disponibilidad. 


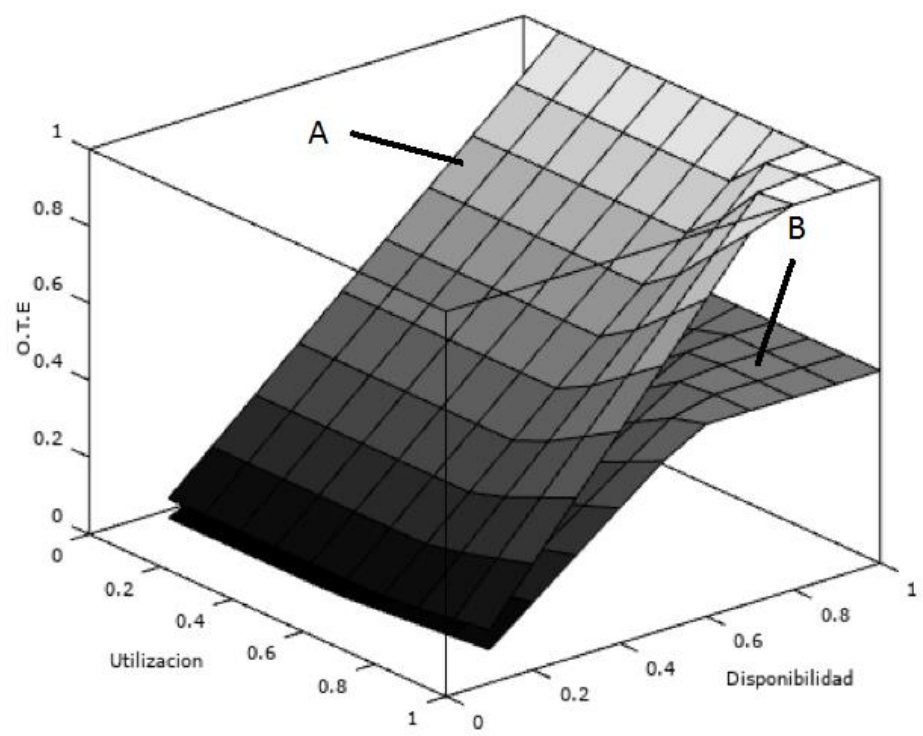

Fig. 2: Sensibilidad en la eficiencia del rendimiento global

Por otro lado, cada equipo presenta diferentes niveles de utilización. Estos niveles de utilización cambian en función de características operativas y están acotados por la disponibilidad que entrega el sector de mantenimiento para el dicho periodo. Si observamos en conjunto ambos parámetros, la sensibilidad y los niveles de utilización, se puede notar que un equipo que presenta una fuerte correlación entre su disponibilidad y los niveles de OTE de sistema y que además ha mostrado altos niveles de utilización le generará una mayor presión al mantenimiento en comparación a otro equipo que, a pesar de tener el mismo nivel de correlación entre su disponibilidad y la OTE, haya mostrado menores niveles de utilización, restándole, en este caso, presión al sector de mantenimiento por más disponibilidad.

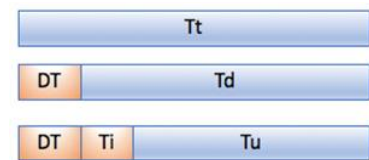

(a)

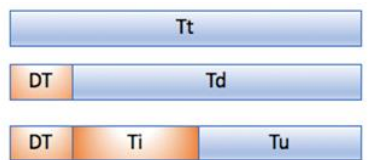

(b)

Fig. 3: Diferentes niveles en la utilización de un equipo

Observe las Figuras $3 a$ y $3 b$, del tiempo total $(T t)$ y descontando el tiempo de parada $(D T)$ queda el tiempo disponible $(T d)$. De ese tiempo, solo parte de él $(T u)$ es efectivamente utilizado, restando un tiempo inutilizado $(T i)$. De esta forma, cuanto menor es el tiempo Ti, más exigido estará el sector de mantenimiento por generar Td. Dicho de otra forma, habrá mayor necesidad de aumentar la Disponibilidad en el equipo del que se hace mención en la figura $3 a$ que en el equipo de la figura $3 b$. Por lo tanto, y considerando dicha situación, se puede construir un diagrama de dispersión con todos los equipos en función de los niveles de correlación (y riesgo) que estos generan entre su disponibilidad y el OTE versus los niveles de utilización que poseen (Figura 4).

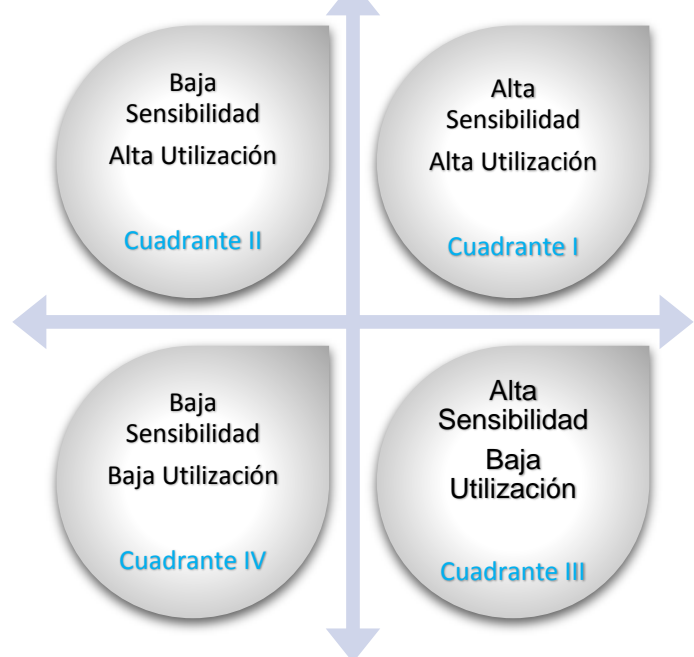

Fig. 4: Diagrama de Dispersión con cuatro zonas de criticidad. 
Cuadrante I: Claramente aquí estarán los equipos que generan un mayor riesgo a la eficiencia del rendimiento global (correlación entre su disponibilidad y el OTE) y tienen un nivel de utilización más alto, deben ser considerados con mayor prioridad que aquellos que no tienen estas características; Cuadrante II: En segundo lugar, y desde el punto de vista de la función mantenimiento, deben ser considerados aquellos equipos que están en el cuadrante II, con menor correlación entre la Disponibilidad y el OTE y con altos niveles de utilización, pues cualquier alteración en su disponibilidad puede redundar en efectos en la eficiencia del rendimiento global. Esto último, en menor grado que aquellos que se encuentran en el cuadrante I; Cuadrante III: Allí se encontrarán los equipos que, teniendo una alta correlación entre su Disponibilidad y el OTE, no han presentado altos niveles de utilización. En estos equipos, la mayor presión por desempeño estará direccionada a aumentar los niveles de utilización, o sea, la presión recaerá en los administradores operacionales, los que tendrán que buscar formas de reducir las pérdidas operacionales y otras detenciones que generen pérdidas de tiempo en el equipo. Aquí podríamos hablar de equipos prioritarios para la gestión de operaciones, no para la gestión del mantenimiento; y Cuadrante IV: Aquí estarán los equipos que no deben ser considerados como prioritarios, pues además de no mostrar una alta correlación (no generan gran impacto) entre sus disponibilidades y el OTE, presentan bajos niveles de utilización, poniendo bajo presión al sector de gestión de operaciones, al igual que los equipos que están en el cuadrante III.

Si se quiere administrar al mantenimiento con una visión de negocio, claramente, se deben priorizar los esfuerzos de mejoría hacia aquellos que generan mayor impacto en el desempeño global del sistema, visto aquí como la correlación entre la disponibilidad y el OTE. Esto último, junto con el análisis de los niveles de utilización, dará indicativos de hacia dónde centrar las atenciones del mantenimiento y los recursos involucrados, promoviendo el uso de ellos donde se pueden recoger la mayor cantidad de buenos resultados a nivel global. La sensibilidad de una variable en relación a la variación de otra, constituye una forma robusta de conocer qué tanto el valor de una variable dependiente podrá variar en función de la variación del valor de una variable independiente. Existen diversos métodos para evaluar la sensibilidad entre los que podemos citar: análisis diferencial o método directo (Krieger et al., 1977), medida de uno a la vez (Yu et al., 1991); diseño factorial (Box et al., 1978; Rose, 1983); indice de sensitividad (Hoffman y Gardner, 1983; Bauer y Hamby, 1991). El indice de sensitividad (IS) se calcula usando la relación (6).

I.S. $=\frac{(\text { Dmax }- \text { Dmin })}{D \max }$

Dmin y Dmax representan, respectivamente, los valores mínimos y máximos de la variable dependiente resultante de la variación de los valores de la variable independiente a lo largo de su intervalo de valores permitidos. Otros métodos usados para realizar estudios de sensibilidad son: Factores de Importancia (Importance Factors) y el Análisis Subjetivo de Sensibilidad (Subjective Sensitivity Analysis) (Downing et al.,1985). En este trabajo, por su simplicidad y nivel de aceptación, se utilizó el indice de sensitividad como parámetro para verificar el grado de variación del OTE al variar la disponibilidad de cada equipo componente del sistema productivo entre los valores de $10 \%$ y $100 \%$ de disponibilidad.

\section{ESTUDIO DE CASO}

Se presenta el caso de una línea de chancado primario existente en una operación minera localizada en los Andes centrales de Chile. En esta Planta se realiza la primera reducción de tamaño del mineral proveniente de la mina, donde el mineral es enviado a la tolva de Traspaso. La Planta tiene una capacidad nominal de 4200 [TPH]. La figura muestra el diagrama de flujo de la planta y la configuración de los principales equipos que forman la planta.

Desde el punto de vista de la configuración, se considera inicialmente que los dos alimentadores de Placas (1 y 2) se encuentran trabajando en paralelo conformando una estación de trabajo que llamaremos Alimentación, que a su vez se encuentra en serie con el resto de los equipos, tal como muestra la figura 6. La tabla 2 muestra las disponibilidades para cada uno de los equipos para 7 meses en que dura el análisis. La tabla 3 muestra las utilizaciones de los mencionados equipos para los siete meses en análisis. Por último, la eficiencia de la calidad se ha medido como la cantidad de producto que sale de la línea en función de la cantidad nominal que se espera que salga de la línea. Las eficiencias de calidad para cada equipo y para cada uno de los siete meses que se analizan son mostradas en la tabla 4. 


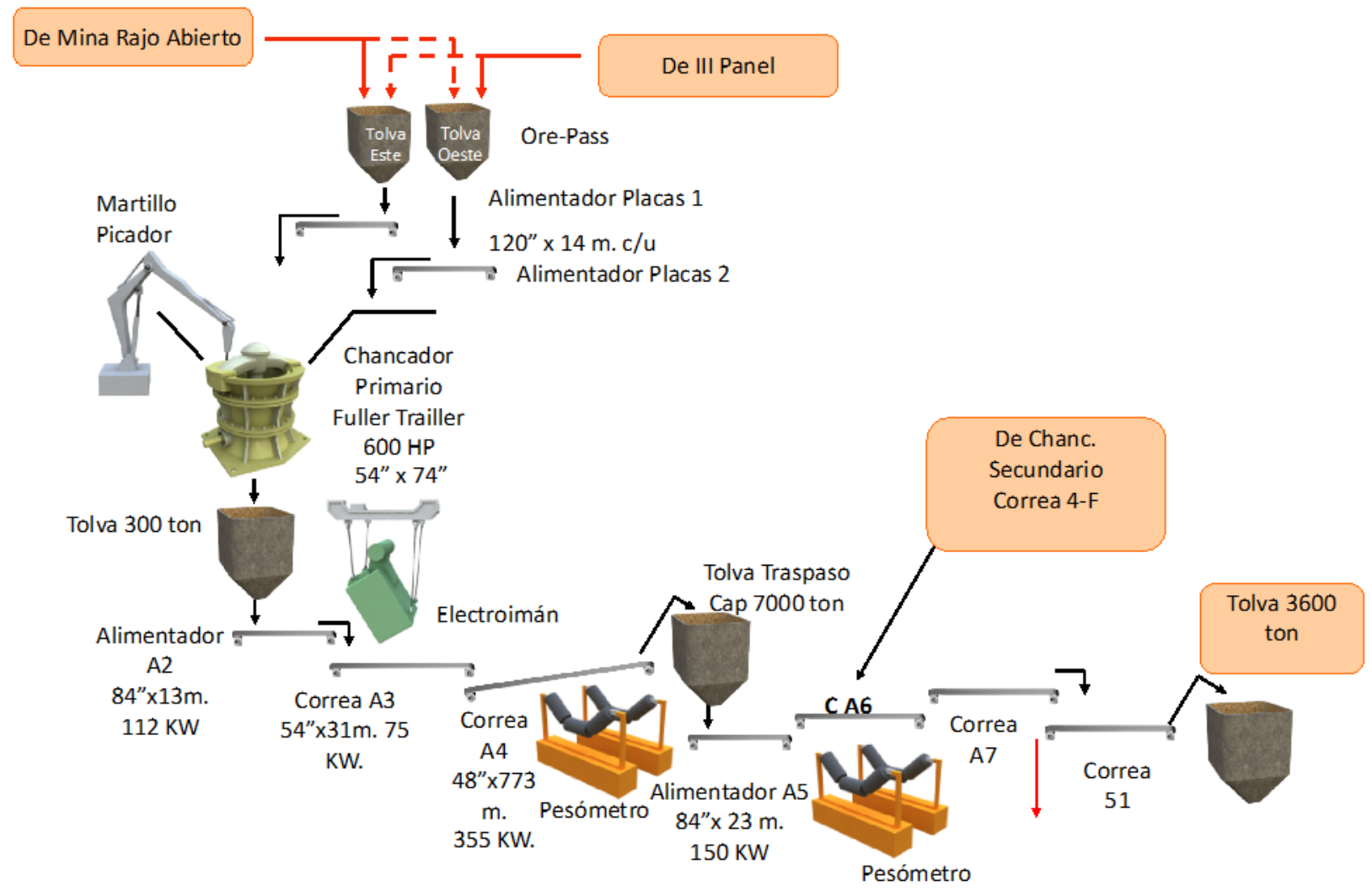

Fig. 5: Diagrama de Flujo de la Planta de Chancado Primario.

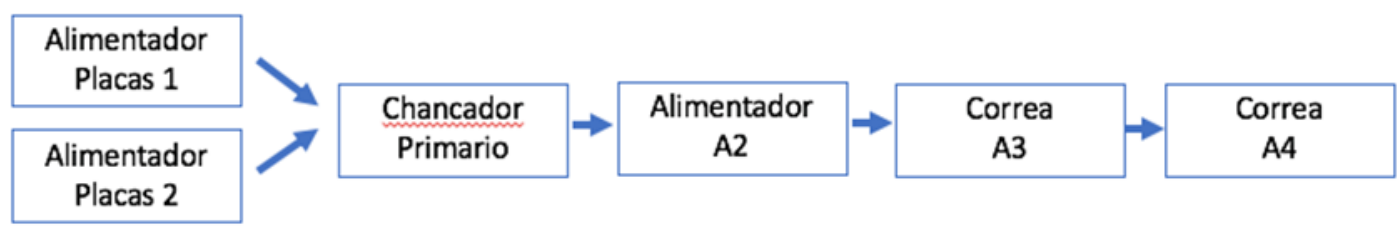

Fig. 6: Configuración global en serie del sistema productivo

Tabla 2: Valores de disponibilidad para cada equipo en los siete meses del análisis.

\begin{tabular}{|l|r|r|r|r|r|r|r|}
\hline & \multicolumn{1}{|c|}{ Mes 1 } & \multicolumn{1}{c|}{ Mes2 } & \multicolumn{1}{c|}{ Mes3 } & \multicolumn{1}{c|}{ Mes4 } & \multicolumn{1}{c|}{ Mes5 } & \multicolumn{1}{c|}{ Mes6 } & \multicolumn{1}{c|}{ Mes7 } \\
\hline Alimentador Placas 1 & $16,88 \%$ & $0,00 \%$ & $0,00 \%$ & $83,04 \%$ & $91,05 \%$ & $97,17 \%$ & $95,98 \%$ \\
\hline Alimentador Placas 2 & $74,81 \%$ & $91,81 \%$ & $93,35 \%$ & $95,76 \%$ & $100,00 \%$ & $93,33 \%$ & $95,54 \%$ \\
\hline Chancador Giratorio & $73,91 \%$ & $90,91 \%$ & $86,17 \%$ & $92,50 \%$ & $93,62 \%$ & $92,25 \%$ & $89,10 \%$ \\
\hline Alimentador A2 & $76,61 \%$ & $92,16 \%$ & $92,43 \%$ & $93,25 \%$ & $93,65 \%$ & $95,37 \%$ & $89,06 \%$ \\
\hline Correa A3 & $77,76 \%$ & $92,40 \%$ & $93,13 \%$ & $93,40 \%$ & $94,26 \%$ & $94,10 \%$ & $87,79 \%$ \\
\hline Correa A4 & $77,33 \%$ & $92,00 \%$ & $92,20 \%$ & $93,40 \%$ & $89,37 \%$ & $93,43 \%$ & $85,09 \%$ \\
\hline
\end{tabular}

Tabla 3: Valores de utilización para cada equipo en los siete meses del análisis.

\begin{tabular}{|l|r|r|r|r|r|r|r|}
\hline & \multicolumn{1}{|c|}{ Mes 1 } & \multicolumn{1}{c|}{ Mes2 } & \multicolumn{1}{c|}{ Mes3 } & \multicolumn{1}{c|}{ Mes4 } & Mes5 & \multicolumn{1}{c|}{ Mes6 } & \multicolumn{1}{c|}{ Mes7 } \\
\hline Alimentador Placas 1 & $4,00 \%$ & $0,00 \%$ & $0,00 \%$ & $60,56 \%$ & $50,22 \%$ & $65,11 \%$ & $60,70 \%$ \\
\hline Alimentador Placas 2 & $56,26 \%$ & $67,72 \%$ & $77,64 \%$ & $67,13 \%$ & $18,57 \%$ & $18,95 \%$ & $11,15 \%$ \\
\hline Chancador Giratorio & $58,87 \%$ & $67,33 \%$ & $68,85 \%$ & $69,63 \%$ & $63,35 \%$ & $65,87 \%$ & $66,81 \%$ \\
\hline Alimentador A2 & $61,37 \%$ & $71,59 \%$ & $78,17 \%$ & $72,84 \%$ & $65,07 \%$ & $70,22 \%$ & $69,27 \%$ \\
\hline Correa A3 & $59,85 \%$ & $72,32 \%$ & $78,19 \%$ & $72,20 \%$ & $67,13 \%$ & $69,78 \%$ & $68,06 \%$ \\
\hline Correa A4 & $63,48 \%$ & $72,18 \%$ & $80,18 \%$ & $72,25 \%$ & $64,99 \%$ & $70,62 \%$ & $64,99 \%$ \\
\hline
\end{tabular}


Tabla 4: Valores de eficiencias de calidad para cada equipo en los siete meses del análisis.

\begin{tabular}{|l|r|r|r|r|r|r|r|}
\hline & Mes 1 & Mes2 & \multicolumn{1}{c|}{ Mes3 } & Mes4 & Mes5 & Mes6 & Mes7 \\
\hline Alimentador Placas 1 & $82,12 \%$ & $87,33 \%$ & $86,08 \%$ & $87,14 \%$ & $89,66 \%$ & $85,56 \%$ & $87,23 \%$ \\
\hline Alimentador Placas 2 & $82,12 \%$ & $87,33 \%$ & $86,08 \%$ & $87,14 \%$ & $89,66 \%$ & $85,56 \%$ & $87,23 \%$ \\
\hline Chancador Giratorio & $82,12 \%$ & $87,33 \%$ & $86,08 \%$ & $87,14 \%$ & $89,66 \%$ & $85,56 \%$ & $87,23 \%$ \\
\hline Alimentador A2 & $82,12 \%$ & $87,33 \%$ & $86,08 \%$ & $87,14 \%$ & $89,66 \%$ & $85,56 \%$ & $87,23 \%$ \\
\hline Correa A3 & $82,12 \%$ & $87,33 \%$ & $86,08 \%$ & $87,14 \%$ & $89,66 \%$ & $85,56 \%$ & $87,23 \%$ \\
\hline Correa A4 & $82,12 \%$ & $87,33 \%$ & $86,08 \%$ & $87,14 \%$ & $89,66 \%$ & $85,56 \%$ & $87,23 \%$ \\
\hline
\end{tabular}

Como resultado de estos valores se calcularon los O.E.E. para cada equipo y se promediaron para realizar el análisis del O.T.E considerando este valor medio. Los valores de OEE promedio para cada equipo se muestran en tabla 5. El valor del O.T.E considerando los siete meses bajo análisis es de 33,10\%. Luego se procedió a realizar cálculos que permitieron determinar cuál equipo presentaba un mayor Índice de Sensibilidad del OTE al variar los niveles de disponibilidad. La tabla 6 muestra los valores de estos índices junto con los valores de Utilización media durante los meses en análisis. A partir de la tabla 6 , se construyó el diagrama de dispersión entre los Índices de Sensibilidad y las Utilizaciones, permitiendo identificar los equipos prioritarios entre los pertenecientes al sistema productivo (Figura 7).

Tabla 5: Valores promedio de O.E.E. para cada equipo

\begin{tabular}{|l|r|}
\hline Alimentador Placas 1 & $32,67 \%$ \\
\hline Alimentador Placas 2 & $40,12 \%$ \\
\hline Chancador Giratorio & $57,06 \%$ \\
\hline Alimentador A2 & $60,41 \%$ \\
\hline Correa A3 & $60,22 \%$ \\
\hline Correa A4 & $60,39 \%$ \\
\hline
\end{tabular}

Tabla 6: Valores promedio de utilización y de los índices de sensibilidad para cada equipo

\begin{tabular}{|l|r|r|}
\hline \multicolumn{1}{|c|}{ Equipo } & Utilización & I.S. \\
\hline Alimentador Placas 1 & $68 \%$ & $30 \%$ \\
\hline Alimentador Placas 2 & $50 \%$ & $44 \%$ \\
\hline Chancador Giratorio & $74 \%$ & $89 \%$ \\
\hline Alimentador A2 & $77 \%$ & $86 \%$ \\
\hline Correa A3 & $77 \%$ & $86 \%$ \\
\hline Correa A4 & $78 \%$ & $0 \%$ \\
\hline
\end{tabular}

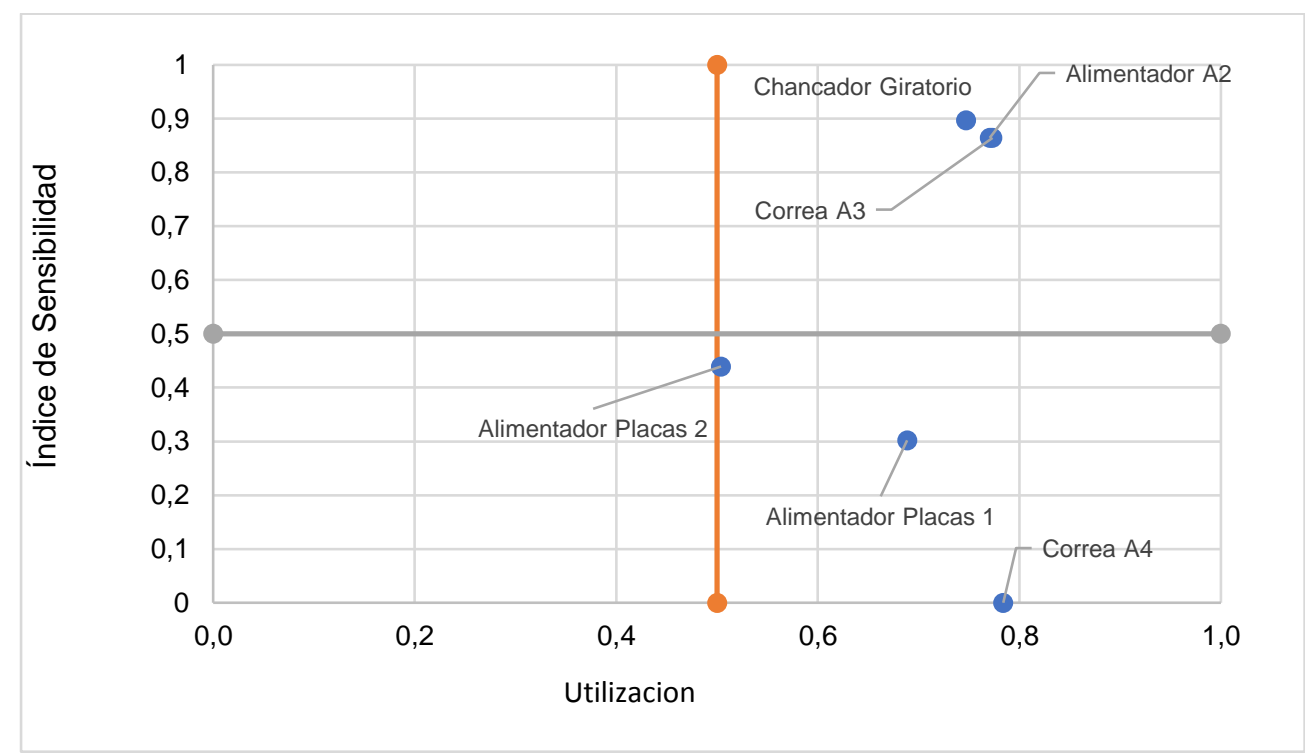

Fig.7: Diagrama de dispersión entre Utilización e Índice de Sensibilidad de cada equipo de la planta de chancado 


\section{ANALISIS DE TENDENCIAS}

Un beneficio adicional de los diagramas de dispersión logarítmicos es que proporcionan un medio útil para visualizar las tendencias al cambio de en las prioridades para el mantenimiento. La tabla 7 muestra la evolución del comportamiento analizados durante un período de tres años consecutivos. La figura 8 muestra el comportamiento en el tiempo de dichos indicadores. Las flechas indican las migraciones de dichos indicadores a lo largo del período de comparación.

Tabla 7: Tendencias de la utilización y del índice de sensibilidad en tres meses del análisis.

\begin{tabular}{|l|l|l|}
\hline Alimentador A2 & Utilización & Índice de Sensibilidad \\
\hline Año 1 & $90 \%$ & $84 \%$ \\
\hline Año 3 & $50 \%$ & $90 \%$ \\
\hline Alimentador Placas & Utilización & Índice de Sensibilidad \\
\hline Año 1 & $40 \%$ & $45 \%$ \\
\hline Año 3 & $90 \%$ & $39 \%$ \\
\hline Chancador Giratorio & Utilización & Índice de Sensibilidad \\
\hline Año 1 & $70 \%$ & $90 \%$ \\
\hline Año 3 & $90 \%$ & $88 \%$ \\
\hline
\end{tabular}

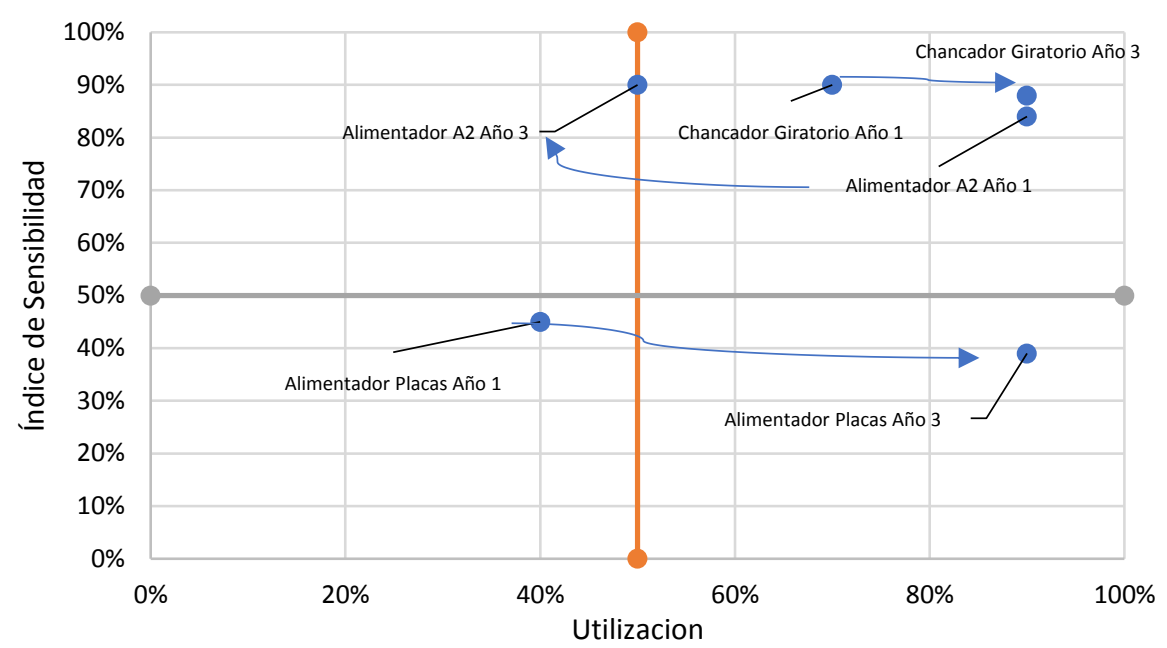

Fig.8: Diagrama de dispersión para análisis de tendencias de tres equipos.

Al observar la figura 8 se pueden observar los siguientes hechos. El caso del Alimentador A2, a lo largo de los tres años de análisis hubo una baja significativa en los niveles de utilización. Esto le restaría prioridad del punto de vista del mantenimiento, pues $50 \%$ de la capacidad disponible no estaría siendo utilizada. Ya en los casos del Alimentador de Placas y del Chancador Giratorio las utilizaciones aumentaron, adicionándole una mayor presión al mantenimiento por generar mayor disponibilidad o al menos, mantenerla, para así incrementar o no perder rendimiento a nivel del sistema productivo. Sin embargo, el nivel de sensibilidad del O.T.E a as variaciones en la sensibilidad en ambos casos, son significativamente diferentes. En el caso del Alimentador de placas, la sensibilidad es menor que en el caso del Chancador Giratorio. O sea, el rendimiento global variará en mayor grado cuando la disponibilidad del Chancador Giratorio sufra variaciones en su disponibilidad. O sea, en este equipo habrá más presión al sector de mantenimiento para mantener o incrementar sus niveles de rendimiento global. Pues los niveles de utilizaciones se están aproximando a los disponibles del equipo.

Como ya fue comentado, cuanto más cerca del origen del diagrama se encuentra un equipo, menos crítico para el sector de mantenimiento será este, pues presentaría un bajo nivel de utilización y baja sensibilidad del OTE a alteraciones en la disponibilidad del activo. Una forma analítica y muy simple de comparar las posiciones de diversos equipos en el diagrama es midiendo la distancia del punto de cada equipo al origen del diagrama $(\Delta)$.

$\Delta=\sqrt{(U t)^{2}+(S . I .)^{2}}$ 
Esta distancia euclidiana $\Delta$ permite clasificar u ordenar dichos equipos en relación con la prioridad que estos deberían tener para el sector de mantenimiento. Observando los valores de la Tabla 7, se calcularon dichas distancias, estas se muestran en la Tabla 8. Aquí el Chancador Giratorio presenta la mayor distancia, seguido por el Alimentador A2 y la Correa A3. Estos equipos requieren de la mayor atención por estar más alejados del origen. Por otro lado, el Alimentador de placas 2 se localiza más cercano al origen, esto hace suponer que es el equipo que presenta una más baja utilización en el periodo y el O.T.E. no es significativamente sensible a alteraciones en la disponibilidad de este.

Tabla 8: Distancias al origen del diagrama de cada uno de los equipos analizados.

\begin{tabular}{|l|l|}
\hline Alimentador Placas 1 & 0,74 \\
\hline Alimentador Placas 2 & 0,67 \\
\hline Chancador Giratorio & 1,16 \\
\hline Alimentador A2 & 1,15 \\
\hline Correa A3 & 1,15 \\
\hline Correa A4 & 0,78 \\
\hline
\end{tabular}

Esta distancia también puede ser analizada en el contexto de su comportamiento en el tiempo. No está demás decir que dos o más equipos podrán presentar la misma distancia al origen, no teniendo los mismos niveles de utilización y de sensibilidad del O.T.E., de ahí la utilidad del diagrama. Esto es, a través del gráfico de dispersión se podrá constatar si el equipo prioritario lo es por un alto nivel de utilización, o por una alta sensibilidad del O.T.E, o por ambas cosas.

El análisis de tendencias en los diagramas de dispersión proporciona un medio visual útil para analizar y controlar las prioridades del mantenimiento desde el punto de vista del OTE. A través de este tipo de análisis, se puede asistir a la preparación de presupuestos de mantenimiento y destinar mayor atención a aquellos equipos que pueden contribuir efectivamente a aumentar el desempeño productivo del sistema. Asimismo, con las debidas atenciones de mantenimiento se podrá evitar o aminorar los perjuicios al desempeño del sistema por una caída en las disponibilidades de dichos equipos.

\section{CONCLUSIONES}

Este trabajo propone una técnica para priorizar equipos de acuerdo con el efecto de la indisponibilidad que presentan y de sus niveles de utilización sobre el rendimiento (a nivel de sistema). Esta técnica representa una herramienta eficaz para evaluar y mostrar cuantitativamente cómo la eficiencia del sistema se ve alterada al variar los valores específicos de disponibilidad de cada equipo. Esto resulta ser interesante pues permiten priorizar los equipos desde una perspectiva de negocio en vez de hacerlo en función de la disponibilidad de cada uno. Así se evita el uso de criterios a nivel de equipo, como se hace al usar los diagramas Jack Knife u otras técnicas basadas en el principio de Pareto. A través del uso de esta técnica se observa la paradoja del equipo que se destaca por los altos niveles de indisponibilidad y que no contribuye necesariamente a la eficiencia a nivel de sistema. La mayor ventaja de la técnica propuesta es que las técnicas existentes no toman en cuenta la configuración del sistema productivo y la forma en que las contribuciones locales se traducen en resultado global (OTE)

La técnica puede ser utilizada para definir las políticas de mantenimiento, destinación de recursos y revisión de decisiones operativas. Como desarrollo futuro, la técnica se puede extender incorporando como tercera dimensión el aspecto de los costos. Esta técnica también admite incorporar la incertidumbre, incluyendo análisis de sensibilidad, midiendo el efecto de largo plazo de políticas de mantenimiento.

\section{REFERENCIAS}

Alsyouf, I., Balanced Scorecard Concept Adapted To Measure Maintenance Performance: A Case Study, in: A.G. Starr and Raj B.K.N. Rao (Eds.), Condition Monitoring and Diagnostic Engineering Management, 227- 234, Elsevier (2001)

Bauer, L.R. y D.M. Hamby, Relative Sensitivities of Existing and Novel Model Parameters in Atmospheric Tritium Dose Estimates, doi.org/10.1093/oxfordjournals.rpd.a081059, Rad. Prot. Dosimetry, 37, 253-260 (1991)

Bezerra Souto Maior, C., M. das Chagas Moura y otros tres autores, Remaining Useful Life Estimation by Empirical Mode Decomposition and Support Vector Machine, doi: 10.1109/TLA.2016.779583, IEEE Lat Am Trans, 14 (11) 46034610, Nov. (2016)

Box, G.E.P., W.G. Hunter y J.S. Hunter, Statistics for Experimenters: An Introduction to Design, Data Analysis, and Model Building, John Wiley \& Sons, New York (1978) 
Crespo Márquez, A., P. Moreu De León, A. Sola Rosique y J.F. Gómez Fernández, Criticality Analysis for Maintenance Purposes: A Study for Complex In-service Engineering Assets, doi.org/10.1002/qre.1769, Qual \& Rel Eng Int, 32 (2), 519-533 (2016)

Crespo Márquez, A., The Maintenance Management Framework. Models and Methods for Complex Systems Maintenance, United Kingdom, Springer (2007)

Durán J.B., Nuevas Tendencias en el Mantenimiento en La Industria Eléctrica, ISSN: 1548-0992 IEEE Lat Am Trans, 1 (1) October (2003)

Espinosa, F. y R. Cea, Diseño de un Programa Computacional para Evaluar la Gestión de Mantenimiento Basado en la Seguridad de Funcionamiento, https://dx.doi.org/10.4067/S0718-07642004000600011, Información Tecnológica, 15(6), 71-78 (2004)

Gasca, M.C., L.L. Camargo y B. Medina, Sistema para Evaluar la Confiabilidad de Equipos Críticos en el Sector Industrial, https://dx.doi.org/10.4067/S0718-07642017000400014, Información Tecnológica, 28(4), 111-124 (2017)

Hoffman, E.O. y R.H. Gardner, Evaluation of Uncertainties in Environmental Radiological Assessment Models, in: Till, J.E., Meyer, H.R. (Eds) Radiological Assessments: a Textbook on Environmental Dose Assessment, Washington, DC, U.S. Nuclear Regulatory Commission, Report No. NUREG/CR-3332 (1983)

Krieger, T.J., C. Durston y D.C. Albright, Statistical Determination of Effective Variables in Sensitivity Analysis, Trans. Am. Nuc. Soc. 28, 515-516 (1977)

Kristjanpoller, F., P. Viveros y A. Crespo Marquez, RAM-C: A novel methodology for evaluating the impact and the criticality of assets over systems with complex logical configurations, Actas del 25th European Safety and Reliability Conference, Zurich, Switzerland (2015)

Lanza, G., J. Stoll, N. Stricker, S. Peters y C. Lorenz, Measuring global production effectiveness, doi.org/10.1016/j.procir.2013.05.006, Procedia CIRP, 7, 31-36 (2013)

Muthiah, K. y S. Huang, Overall throughput effectiveness (OTE) metric for factory-level performance monitoring and bottleneck detection, doi.org/10.1080/00207540600786731, Int. J. Prod Res, 45, No. 20, S.;1; 4753 - S.;1; 4769 (2007)

Muthiah, K., S. Huang y S. Mahadevan, Automating factory performance diagnostics using overall throughput effectiveness (OTE) metric., doi.org/10.1007/s00170-006-0891-x, Int. J. Adv. Mfing, Tech., 36 (7-8), 811-824 (2008)

Nakajima, S., Total Productive Maintenance - Introduction to TPM, Productivity Press 1988, ISBN 0-915299-23-2 (1988)

Nelson R. y S.M. Kannan, Overall Process Effectiveness (OPE) model for the tyre manufacturing industry, Department of mechanical engineering, International conference on OPE Velanmmal College of Engineering \& Technology, Madurai625009, India (2010)

Parra, C. y A. Crespo, Técnicas de Ingeniería de Mantenimiento y Fiabilidad aplicadas en el proceso de Gestión de Activos. Nota técnica 5: Métodos de Análisis de criticidad y jerarquización de activos, Asociación para el desarrollo de la ingeniería de mantenimiento, España (2012)

Pascual, R., D. Godoy y D.M. Louit, Throughput centered prioritization of machines in transfer lines, doi.org/10.1016/j.ress.2011.05.006, Rel Eng \& Syst Safety, 96, 1396-1401 (2011)

Pascual, R., R. Madariaga, G. Santelices, D. Godoy y E.L. Droguett, A Structured Methodology to Optimise Throughput of Production Lines, doi.org/10.1080/17480930.2014.962235, Int. J. Mining, Reclam and Env., 30 (1) 25-36 (2016)

Rose, K.A., A Simulation Comparison and Evaluation of Parameter Sensitivity Methods Applicable to Large Models, in: Analysis of Ecological Systems: State-of-the-art in Ecological Modelling (Lauenroth, G.V. Skogerboe, M. Flug Eds.), Proceedings of a meeting at Colorado State University, May 24-28, 1982, Elsevier, New York (1983)

Silva, M.D.B., C. Mattos Affonso, K.C.S Cavalcante y F.E.L. Picanco, Productivity Estimation to Diagnosis Support in Bauxite Open-pit Mine Using Fuzzy Logic, doi: 10.1109/TLA.2014.7014533 IEEE Lat Am Trans, 12 (8), 1595-601, Dec. (2014)

Yu, C., Cheng, J-J. y A'J. Zielen, Sensitivity Analysis of the RESRAD, a Dose Assessment Code, ISSN 0003-018X Trans. Am. Nuc. Soc. 64; 73-74 (1991) 
